\title{
The Effect of Fat Emulsion Intralipid 20\% in Reproductive Outcome for Patients With Multiple Implantation Failure
}

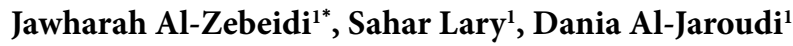

\begin{abstract}
Objectives: The aim of this study is to evaluate the effect of fat emulsion intralipid $20 \%$ on reproductive outcome of patients with multiple implantation failure (MIF).

Materials and Methods: A retrospective study of 30 women with MIF who received intralipid 20\% with in vitro fertilization, intracytoplasmic sperm injection (IVF-ICSI). The treatment cycle was conducted in reproductive endocrine and infertility medicine department (REIMD) at Women Specialized Hospital, King Fahad Medical City (KFMC), from January 2015 to December 2016. Results: Thirteen of 30 women (43.3\%) had a positive pregnancy test and 17 (56.7\%) did not. Pregnancy rate showed no statistically significant difference with the use of long protocol in comparison to short antagonist protocol. Use of intralipid $20 \%$ did not affect the embryo grading or yield the higher number of frozen embryos.

Conclusions: MIF is a challenging situation in reproductive medicine. Intralipid 20\% might be an effective treatment for patients with MIF.

Keywords: Fat emulsion, Intracytoplasmic intralipid, Multiple implantation failure, Pregnancy rate, Sperm injection rate.
\end{abstract}

\section{Introduction}

Multiple implantation failure (MIF) is described as implantation failure in spite of transferring good quality embryos following several in vitro fertilization (IVF) treatment cycles (1). Implantation is a complicated process that requires healthy embryos with receptive endometrium. Many factors can lead to implantation failure due to either maternal or embryonic factors. Uterine anatomy, thrombophilia, immunological factors, and a non-receptive endometrium are amongst the maternal factors and genetic or lab related are amongst the embryonic factors (1).

The patient with repeated implantation failure is a challenge for the infertility specialists. Age remains the most important variable influencing outcome in assisted reproduction (2-4). Advanced maternal age manifests its effects on the clinical in vitro fertilization embryo transfer (IVF-ET) procedure that is due to the variable effects on the pattern of ovarian response and on the reduction of implantation efficiency and due to an increased spontaneous abortion rate (2-4).

Impaired uterine receptivity leads to implantation failure in assisted reproduction (2-4). Thrombophilia and abnormal immunological response could result in a non-receptive endometrium (2-4) and eventually in implantation failure.
Investigation about recurrent implantation failure includes tests for inherited and acquired thrombophilias and with immunological causes. Management should be individualized according to the different etiologies and empirical treatments with low molecular weight heparin (LMWH). Aspirin or corticosteroids are not effective for women with RIF whose test results are negative in thrombophilic tests (2-4). If leukocyte antigen dissimilarity is proven immunologically, treatment with intravenous immunoglobulin (IVIG) might be helpful; however, treatment with intralipid infusion in the presence of increased natural killer cytotoxic activity could be helpful too but is still controversial. Data supporting this practice is conflicting.

The suggested therapy in recurrent implantation failure is intralipid, which is a fat emulsion containing soybean oil, glycerin, egg phospholipids, glycerol, and water. It provides essential fatty acids, linoleic acid, omega-3 and 6 fatty acids, and alpha-linolenic acid (5). Intralipid suppresses natural killer cell activity $(\mathrm{NKa})$ is similar to the effect of IVIG (5).

Intralipid is a milky solution, originally used to provide energy to the patients who could not eat (6) and it is made of purified soybean oil and egg phospholipids. Intralipid has been initially used for parenteral nutrition in patients who are unable to take food orally (6). The mechanism of 
action of the parenteral fat emulsions is the accumulation in macrophages and impairment of their various functions (6). Although the exact immune mechanism by which intralipid acts is not clear, the soybean oil may be the active component that inhibits pro-inflammatory mediators, specifically type 1 T-helper cells (7).

Kalfarentzos et al studied the differences between fat emulsions, intralipid 10\%, and intralipid 30\% and compared the resulting plasma levels of different lipid components. There were no differences between them. However, intralipid $30 \%$ had more lipid components in comparison to intralipid $10 \%$. The fat emulsion with greater concentration of triglyceride has been found to be safe and is used for sick patients requiring total parenteral nutrition (7).

Imbalance of the immune system during implantation or pregnancy may lead to implantation failure or miscarriage and therefore the usage of immunosuppressive or immunomodulatory agents can prevent immunological occurrence (6).

Intralipids have immunosuppressive effects (6) and thus the intravenous intralipid injection affects the proliferation and function of immune cells including the lymphocytes (8) and by inhibiting the IL-2, it decreases the activation signals for $\mathrm{T}$ and $\mathrm{B}$ lymphocyte (9).

When used in ICSI cycles with patients with RIF, intravenous intralipid 20\% infusion improves the clinical pregnancy, the implantation, and the live birth rate (10).

MIF is a distressing condition affecting couples. Immunotherapeutic agents such as immunomodulators and immunosuppressive are new factors but they have been used for managing patients with MIF and have been found to eliminate the damaging reactions against the fetus $(8,9)$. Few studies have been conducted to assess intralipid use in patients undergoing assisted reproductive technologies (ART).

The aim of this study is to evaluate the effect of fat emulsion intralipid 20\% on reproductive outcomes of patients with MIF undergoing ART.

\section{Materials and Methods}

Thirty women with MIF participated in this study. They were at reproductive endocrine and infertility medicine department (REIMD) at women specialized hospital, King Fahad Medical City (KFMC) from January 2015 to December 2016. All charts of women with 3 or more unsuccessful IVF cycles who received $20 \%$ intralipid fat emulsion, age less than 45 , and normal uterine cavity were reviewed. The charts were excluded from our analysis if any of the following conditions were recorded in the chart: a patient with medical condition contraindicating the use of intralipid infusion, uterine fibroid, endometrial polyp, endometriosis and hydrosalpinx, intrauterine adhesion and uterine anomalies, deficiency of protein $\mathrm{C}$, protein S, factor V Leiden, antiphospholipid syndrome (lupus anticoagulant), anticardiolipin antibodies (immunoglobulin $\mathrm{G}$ or $\mathrm{M}$ ) $[\operatorname{IgG}$ or $\operatorname{IgM}]$ ), and other recognized thrombophilic conditions.

\section{Statistical Analysis}

MS Excel 2010 and SPSS 22.0 software were used for data analysis. Categorical variables were expressed as frequencies and percentages and were analyzed using chi-square tests. Metric data was presented as the mean, +/- standard deviation, and analyzed using independent sample $t$ tests. All statistical tests were 2-tailed. The hypotheses were tested based on the $P$ value, where $P$ less than 0.05 was considered as statistically significant.

\section{Results}

Thirty patients were included in the study, 27 of them underwent IVF -ICSI cycle and 3 underwent frozen embryo transfer (FET) cycles. The latter was evaluated for the effect of administration of intravenous intralipid on pregnancy outcome.

Patient demographics and clinical characteristics were demonstrated in Table 1 . The identified causes of infertility were tubal factor in $(16.7 \%, \mathrm{n}=5)$, polycystic ovary $(\mathrm{PCO})$

Table 1. Basic Demographic and Clinical Characteristics of the Patients $(n=30)$

\begin{tabular}{|c|c|c|}
\hline Variables & Characteristics & No. (\%) \\
\hline \multirow{2}{*}{ Female occupation } & House wife & $26(86.7)$ \\
\hline & Professionals & $4(13.3)$ \\
\hline \multirow{2}{*}{ Husband occupation } & Employed & $10(33.3)$ \\
\hline & Unemployed & $20(66.7)$ \\
\hline \multirow{2}{*}{ Hysteroscopy finding } & Yes & $17(56.7)$ \\
\hline & No & $13(43.3)$ \\
\hline \multirow{2}{*}{ RIF } & $\geq 3$ (Yes) & $30(100)$ \\
\hline & $<3$ (No) & $0(0)$ \\
\hline \multirow{3}{*}{ HSG findings } & Normal & $13(43.3)$ \\
\hline & Bilateral block & 5 (16.7) \\
\hline & Unilateral block & $12(40.0)$ \\
\hline \multirow{7}{*}{ Diagnosis } & Tubal & $5(16.7)$ \\
\hline & PCOS & $4(13.3)$ \\
\hline & Male & $10(33.3)$ \\
\hline & Anovulatory & $2(6.6)$ \\
\hline & Unexplaned & $4(13.3)$ \\
\hline & Multiple & $4(13.3)$ \\
\hline & Other & $1(3.3)$ \\
\hline \multirow{2}{*}{ PCO } & Yes & $4(13.3)$ \\
\hline & No & $26(86.7)$ \\
\hline \multirow{2}{*}{ Male factor } & Yes & $10(33.3)$ \\
\hline & No & $20(66.7)$ \\
\hline \multirow{3}{*}{ Protocol } & Antagonist & $21(70.0)$ \\
\hline & Long & $6(20.0)$ \\
\hline & FET & $3(10.0)$ \\
\hline \multirow{4}{*}{ Drug } & Cetrotide & $21(70.0)$ \\
\hline & Lupron & $5(16.7)$ \\
\hline & Estradiol valerate & $3(10.0)$ \\
\hline & GnRH agonist (Decapeptyl) & $1(3.3)$ \\
\hline \multirow{4}{*}{ Total AFC } & $\leq 3$ & $0(0.0)$ \\
\hline & $4-8$ & $9(30.0)$ \\
\hline & $9-12$ & $5(16.7)$ \\
\hline & $>12$ & $16(53.3)$ \\
\hline
\end{tabular}


Table 1. Continued

\begin{tabular}{|c|c|c|}
\hline \multirow{4}{*}{ Stimulation drug } & Gonal F (r-FSH) & $12(40.0)$ \\
\hline & Merional (HMG) & $7(23.3)$ \\
\hline & $\begin{array}{l}\text { Gonal F and lutropin alpha } \\
\text { (luveris) }\end{array}$ & $8(26.7)$ \\
\hline & Estradiol valerate & $3(10.0)$ \\
\hline \multirow{4}{*}{ Therapy pattern } & Gonal F (r-FSH) & $12(40.0)$ \\
\hline & Merional (HMG) & $7(23.3)$ \\
\hline & $\begin{array}{l}\text { Combined therapy (GonalF }+ \\
\text { HMG) }\end{array}$ & $8(26.7)$ \\
\hline & Frozen embryos & $3(10.0)$ \\
\hline \multirow{3}{*}{ Trigger drug } & HCG (Pregnyl) & $27(90.0)$ \\
\hline & Other & $0(0.0)$ \\
\hline & FET & $3(10.0)$ \\
\hline \multirow{2}{*}{$\begin{array}{l}\text { Range of mature } \\
\text { oocytes (MII) }\end{array}$} & $\begin{array}{l}<3 \text { (MII not good for } \\
\text { pregnancy) }\end{array}$ & $9(30.0)$ \\
\hline & $\geq 3$ (MII good for pregnancy) & $21(70.0)$ \\
\hline \multirow{4}{*}{ Grades } & G1 & $12(40.0)$ \\
\hline & $\mathrm{G} 2$ & $7(23.3)$ \\
\hline & G3 & $1(3.3)$ \\
\hline & $\mathrm{G} 1+\mathrm{G} 2$ & $10(33.3)$ \\
\hline \multirow{2}{*}{ Frozen embryos } & $\leq 2$ & $23(76.7)$ \\
\hline & $>2$ & $7(23.3)$ \\
\hline \multirow{2}{*}{$\begin{array}{l}\text { Range of the number } \\
\text { of embryo transfers }\end{array}$} & 1 & $6(20.0)$ \\
\hline & 2 & $24(80.0)$ \\
\hline \multirow{2}{*}{ Range sperm count } & $\leq 15$ million (oligospermia) & $6(20.0)$ \\
\hline & >15 million (normal) & $24(80.0)$ \\
\hline \multirow{2}{*}{ Motility range } & $<32 \%$ & $8(26.7)$ \\
\hline & $\geq 32 \%$ & $22(73.3)$ \\
\hline \multirow{2}{*}{ Morphology range } & $<4 \%$ & $6(20.0)$ \\
\hline & $\geq 4 \%$ & $24(80.0)$ \\
\hline \multirow{2}{*}{ Outcome } & Non-pregnant & $17(56.7)$ \\
\hline & Pregnant & $13(43.3)$ \\
\hline
\end{tabular}

RIF, repeated implantation failure; HSG, hysterosalpingogram; PCO, polycystic ovaries; AFC, antral follicle count; PCOS, Polycystic Ovarian Syndrome.

(13.3\%, $\mathrm{n}=4)$, male $(33.3 \%, \mathrm{n}=10)$, anovulatory $(6.6 \%$, $\mathrm{n}=2)$, unexplained $(13.3 \%, \mathrm{n}=4)$, multiple $(13.3, \mathrm{n}=4)$, and other ones such as endometriosis and fibroid (3.3\%, $\mathrm{n}=1$ ). Six patients started with long protocol, 21 of them with antagonist protocol, and 3 with FET cycle. Higher pregnancy rate was found with the use of long protocol in comparison to the short antagonist protocol. Use of intralipid $20 \%$ did not affect embryo grading or yield higher number of frozen embryos.

Table 2 shows the descriptive analysis and laboratory testing of study parameters. Median age for wife was 37 years which ranges from 31 to 45 years, median age of husband was 40 years within the range of 31-60 years, and median duration of infertility was 8 years ranges from 3 to18 years. Median follicle-stimulating hormone (FSH) level was $5.72 \mathrm{IU} / \mathrm{L}$, luteinizing hormone (LH) level was $6.35 \mathrm{IU} / \mathrm{L}$, prolactin level was $254 \mathrm{mIU} / \mathrm{L}$, and thyroid stimulating hormone (TSH) level was $2.23 \mathrm{mIU} / \mathrm{L}$. The median body mass index (BMI) of patients was $29.1 \mathrm{~kg} / \mathrm{m}^{2}$.

Table 3 demonstrates the impact and association of the parameters with pregnancy outcomes.
Table 4 demonstrates the pregnancy rate in the agonist group $4(66.7 \%)$ ] and in the antagonist group (8 [33.1\%]) with no statistical significance. Applying intralipid 20\% did not affect the embryo grading or yield higher number of frozen embryos.

Pregnancy rate with the use of intralipid intravenous infusion was achieved in $43.3 \%$ of the patients and $56.6 \%$ did not get pregnant. There was no significant statistical difference in pregnancy outcome in relation to measured hormonal profiles (Table 4).

\section{Discussion}

The infusion of $20 \%$ intralipid solution has been studied with results showing better outcomes in women with RIF (5). In a non-randomized trial on patients with MIF who had an elevated T helper-1 (TH1) cytokine, a 50\% pregnancy rate and $46 \%$ clinical pregnancy rate were reported (5). The intralipid infusion was given between days 4 and 9 of the ovarian stimulation and was re-given within 7 days of a positive pregnancy test. However, the beneficial effects of the infusion, have shown to be decreased with further treatment cycles. The most amount of reduction in clinical pregnancy rates was obvious in the third IVF treatment cycle and hence immunological tests should be considered in those cases (6).

Clark in 1994 concluded that in a clinical trial intralipid had been successful in treating patients with recurrent miscarriages. It was found that both IVIG and intralipid could suppress NK cell cytotoxicity with equal efficacy in a in vitro assay that made the intralipid a cost-effective option (11). In a more recent study that was published in 2013, impaired endometrial receptivity was assessed and the beneficial effect of intralipid infusion in the presence of increased natural killer cell cytotoxic activity in patients with recurrent implantation failure was identified (12).

Another study showed intralipid suppresses in vivo abnormal NK-cell functional activity and implied that Intralipid can modulate abnormal NK activity in women with reproductive failure (10). In 2016, AbdolmohammadiVahid et al recommended the use of immunosuppressive or immunomodulator agents since the imbalance of the immune system during implantation or pregnancy may lead to implantation failure or miscarriage (13). Thus using intralipid with its inhibitory immunological effect may improve pregnancy outcomes (13).

In our study pregnancy rate with the use of intralipid infusion showed no statistical significance between both groups.

Other studies in the literature have shown no difference in reproductive outcomes in women with recurrent implantation failure who received intravenous immunoglobulin or intralipid. One of them is a casecontrol study that was terminated because of their initial data that reported no pregnancies among the intralipid group compared to untreated control group (14). Many 
Table 2. Descriptive Analysis, Laboratory Testing of Study Parameters

\begin{tabular}{lllll}
\hline & Minimum & Maximum & Median & Mean \pm S.D \\
\hline Female age & 31.00 & 45.00 & 37.0000 & $36.5 \pm 0.65$ \\
Husband age & 31 & 60 & 40.00 & $40.9 \pm 1.06$ \\
Years of infertility & 3.00 & 18.00 & 8.0000 & $9.13 \pm 0.85$ \\
FSH & 1 & 18 & 5.72 & $6.57 \pm 0.62$ \\
LH & 2.00 & 18.00 & 6.3500 & $6.88 \pm 0.58$ \\
Estradiol & 33.47 & 771.00 & 183.5000 & $216.31 \pm 28.16$ \\
Prolactin & 1.32 & 731.20 & 254.0000 & $277.96 \pm 30.55$ \\
Thyroid stimulating hormone (TSH) & .01 & 21.04 & 2.2350 & $2.98 \pm 0.68$ \\
T4 & 10.90 & 23.20 & 14.9000 & $15.14 \pm 0.49$ \\
Vitamin D & 9.30 & 110.40 & 24.6950 & $39.96 \pm 5.94$ \\
Body mass index (BMI) & 20.70 & 37.70 & 29.1000 & $29.24 \pm 0.76$ \\
Dose follicle stimulating hormone (r-FSH) (IU) & 112.5 & 450 & 168.75 & $167.5 \pm 26.47$ \\
Dose of human menopausal gonadotropins (HMG) (mg) & 75 & 450 & 37.50 & $92.5 \pm 20.24$ \\
Total Dose (r-FSH) (IU) & 1025.00 & 4050.00 & 1725.0000 & $1611.67 \pm 251.1$ \\
Total Dose (HMG) (IU) & 825 & 6300.00 & 412.5000 & $955 \pm 246.76$ \\
Sperm Count(million) & 0.75 & 435.00 & 115.0000 & $126.28 \pm 20.87$ \\
B-Human Chorionic Gonadotropin (B-HCG) Test Results & 0.00 & 3627.00 & 2.0700 & $193.66 \pm 122.15$ \\
\hline
\end{tabular}

Table 3. Impact and Association Among Pregnancy Outcome and Other Parameters

\begin{tabular}{|c|c|c|c|c|c|}
\hline & & $\begin{array}{l}\text { Non-Pregnant } \\
\text { No. (\%) }\end{array}$ & $\begin{array}{l}\text { Pregnant } \\
\text { No. (\%) }\end{array}$ & OR $[95 \% \mathrm{Cl}]$ & $P$ Value \\
\hline \multirow{2}{*}{ Hysteroscopy finding } & Yes & $10(58.8)$ & $7(53.8)$ & \multirow{2}{*}{$1.22[0.285-5.255]$} & \multirow{2}{*}{0.785} \\
\hline & No & $7(41.2)$ & $6(46.2)$ & & \\
\hline \multirow{3}{*}{ Hysterosalpingogram findings } & Normal & $8(47.1)$ & $5(38.5)$ & $1.42[0.328-6.174]$ & 0.638 \\
\hline & Bilateral block & $4(23.5)$ & $1(7.7)$ & $3.69[0.36-37.858]$ & 0.249 \\
\hline & Unilateral block & $5(29.4)$ & $7(53.8)$ & $0.36[0.079-1.615]$ & 0.176 \\
\hline \multirow{2}{*}{ Polycystic ovaries } & Yes & $1(5.9)$ & $3(23.1)$ & \multirow{2}{*}{$0.21[0.019-2.29]$} & \multirow{2}{*}{0.170} \\
\hline & No & $16(94.1)$ & $10(76.9)$ & & \\
\hline \multirow{2}{*}{ Male factor } & Yes & $5(29.4)$ & $5(38.5)$ & \multirow{2}{*}{$0.67[0.145-3.075]$} & \multirow{2}{*}{0.602} \\
\hline & No & $12(70.6)$ & $8(61.5)$ & & \\
\hline \multirow{3}{*}{ Protocol } & Antagonist & $13(76.5)$ & $8(61.5)$ & $2.03[0.417-9.887]$ & 0.376 \\
\hline & Long & $2(11.8)$ & $4(30.8)$ & $0.3[0.045-1.982]$ & 0.197 \\
\hline & Frozen embryo transfer & $2(11.8)$ & $1(7.7)$ & $1.6[0.129-19.839]$ & 0.713 \\
\hline \multirow{4}{*}{ Drugs } & GnRH antagonist-Cetrolix (Cetrotide) & $13(76.5)$ & $8(61.5)$ & $2.03[0.417-9.887]$ & 0.376 \\
\hline & Leuprolide & $2(11.8)$ & $3(23.1)$ & $0.44[0.063-3.155]$ & 0.410 \\
\hline & Estradiol Valerate & $2(11.8)$ & $1(7.7)$ & $1.6[0.129-19.839]$ & 0.713 \\
\hline & GnRH agonist (Decapeptyl) & $0(0.0)$ & $1(7.7)$ & $0.35[0.011-11.392]$ & 0.245 \\
\hline \multirow{3}{*}{ Antral follicular count (AFC) } & $4-8$ & $6(35.3)$ & $3(23.1)$ & $1.82[0.357-9.272]$ & 0.469 \\
\hline & $9-12$ & $2(11.8)$ & $3(23.1)$ & $0.44[0.063-3.155]$ & 0.410 \\
\hline & $>12$ & $9(52.9)$ & $7(53.8)$ & $0.96[0.227-4.102]$ & 0.961 \\
\hline \multirow{4}{*}{ Stimulation drug } & r-Follicle Stimulating Hormone (r-FSH) & $7(41.2)$ & $5(38.5)$ & $1.12[0.256-4.905]$ & 0.880 \\
\hline & Human menopausal gonadotropins (HMG) & $5(29.4)$ & $2(15.4)$ & $2.29[0.367-14.323]$ & 0.368 \\
\hline & r-FSH and Lutropin alpha & $3(17.6)$ & $5(38.5)$ & $0.34[0.064-1.829]$ & 0.201 \\
\hline & Estradiol valerate & $2(11.8)$ & $1(7.7)$ & $1.6[0.129-19.839]$ & 0.713 \\
\hline \multirow{4}{*}{ Therapy pattern } & r-Follicle stimulating hormone ( $r-F S H)$ & $7(41.2)$ & $5(38.5)$ & $1.12[0.256-4.905]$ & 0.880 \\
\hline & Human menopausal gonadotropins (HMG) & $5(29.4)$ & $2(15.4)$ & $2.29[0.367-14.323]$ & 0.368 \\
\hline & Combined Therapy (r-FSH + HMG) & $3(17.6)$ & $5(38.5)$ & $0.34[0.064-1.829]$ & 0.201 \\
\hline & Forzen embryos & $2(11.8)$ & $1(7.7)$ & $1.6[0.129-19.839]$ & 0.713 \\
\hline \multirow{2}{*}{ Trigger drug } & Human chorionic gonadotropins (HCG-Pregnyl) & $15(88.2)$ & $12(92.3)$ & \multirow{2}{*}{$0.63[0.05-7.75]$} & \multirow{2}{*}{0.713} \\
\hline & Frozen embryo transfer (FET) & $2(11.8)$ & $1(7.7)$ & & \\
\hline
\end{tabular}




\begin{tabular}{|c|c|c|c|c|c|}
\hline \multirow{2}{*}{ Mature oocytes (MII) } & $<3$ (MII not good for pregnancy) & $6(35.3)$ & $3(23.1)$ & \multirow{2}{*}{$1.82[0.357-9.272]$} & \multirow{2}{*}{0.469} \\
\hline & $\geq 3$ (MIl good for pregnancy) & $11(64.7)$ & $10(76.9)$ & & \\
\hline \multirow{4}{*}{ Embryo grades } & G1 & $6(35.3)$ & $6(46.2)$ & $0.64[0.145-2.784]$ & 0.547 \\
\hline & $\mathrm{G} 2$ & $5(29.4)$ & $2(15.4)$ & $2.29[0.367-14.323]$ & 0.368 \\
\hline & G3 & $1(5.9)$ & $0(0.0)$ & $1.63[0.05-52.369]$ & 0.374 \\
\hline & $\mathrm{G} 1+\mathrm{G} 2$ & $5(29.4)$ & $5(38.5)$ & $0.67[0.145-3.075]$ & 0.602 \\
\hline \multirow{2}{*}{ Frozen embryos } & $\leq 2$ & $13(76.5)$ & 10 (76.9) & \multirow{2}{*}{$0.98[0.177-5.385]$} & \multirow{2}{*}{0.997} \\
\hline & $>2$ & $4(23.5)$ & $3(23.1)$ & & \\
\hline \multirow{2}{*}{ Number of embryo transfers } & 1 & $3(17.6)$ & $3(23.1)$ & \multirow{2}{*}{$0.71[0.119-4.297]$} & \multirow{2}{*}{0.713} \\
\hline & 2 & $14(82.4)$ & $10(76.9)$ & & \\
\hline \multirow{2}{*}{ Sperm count } & $\leq 15$ million (Oligospermia) & $1(5.9)$ & $7(53.8)$ & \multirow{2}{*}{$0.05[0.005-0.532]$} & \multirow{2}{*}{$* 0.003$} \\
\hline & $>15$ million (Normal) & $16(94.1)$ & $6(46.2)$ & & \\
\hline \multirow{2}{*}{ Motility range } & $<32 \%$ & $4(23.5)$ & $4(30.8)$ & \multirow{2}{*}{$0.69[0.136-3.519]$} & \multirow{2}{*}{0.657} \\
\hline & $\geq 32 \%$ & $13(76.5)$ & $9(69.2)$ & & \\
\hline \multirow{2}{*}{ Morphology range } & $<4 \%$ & $3(17.6)$ & $3(23.1)$ & \multirow{2}{*}{$0.71[0.119-4.297]$} & \multirow{2}{*}{0.713} \\
\hline & $\geq 4 \%$ & $14(82.4)$ & $10(76.9)$ & & \\
\hline
\end{tabular}

Table 4. Relationship Between Laboratory Test Among Pregnancy Outcome

\begin{tabular}{|c|c|c|c|c|c|}
\hline & & $\begin{array}{c}\text { Non-Pregnant } \\
\text { No. (\%) }\end{array}$ & $\begin{array}{c}\text { Pregnant } \\
\text { No. (\%) }\end{array}$ & OR $[95 \% \mathrm{Cl}]$ & $P$ Value \\
\hline \multirow{2}{*}{ Follicular stimulating hormone (FSH), IU/L } & Abnormal & $1(5.9)$ & $2(15.4)$ & \multirow{2}{*}{$0.34[0.028-4.273]$} & \multirow{2}{*}{0.390} \\
\hline & Normal & $16(94.1)$ & $11(84.6)$ & & \\
\hline \multirow{2}{*}{ Luteinizing hormone (LH), IU/L } & Abnormal & $0(0.0)$ & $1(7.7)$ & \multirow{2}{*}{$0.35[0.011-11.392]$} & \multirow{2}{*}{0.554} \\
\hline & Normal & $17(100.0)$ & $12(92.3)$ & & \\
\hline \multirow{2}{*}{ Estradiol, pg/mL } & Abnormal & $7(41.2)$ & $5(38.5)$ & \multirow{2}{*}{$1.12[0.256-4.905]$} & \multirow{2}{*}{0.880} \\
\hline & Normal & $10(58.8)$ & $8(61.5)$ & & \\
\hline \multirow{2}{*}{ Prolactin, mIU/L } & Abnormal & $9(52.9)$ & $3(25.0)$ & \multirow{2}{*}{$3.75[0.754-18.642]$} & \multirow{2}{*}{0.098} \\
\hline & Normal & $8(47.1)$ & $9(75.0)$ & & \\
\hline \multirow{2}{*}{ Thyroid stimulating hormone (TSH), mIU/L } & Abnormal & $3(17.6)$ & $4(30.8)$ & \multirow{2}{*}{$0.48[0.087-2.68]$} & \multirow{2}{*}{0.400} \\
\hline & Normal & $14(82.4)$ & $9(69.2)$ & & \\
\hline \multirow{2}{*}{$\mathrm{T} 4, \mathrm{ug} / \mathrm{dL}$} & Abnormal & $0(0.0)$ & $3(25.0)$ & \multirow{2}{*}{$0.1[0.004-2.164]$} & \multirow{2}{*}{0.089} \\
\hline & Normal & $17(100.0)$ & $9(75.0)$ & & \\
\hline \multirow{2}{*}{ Vitamin $\mathrm{D}, \mathrm{ng} / \mathrm{mL}$} & Abnormal & $14(87.5)$ & $8(80.0)$ & \multirow{2}{*}{$2.92[0.547-15.561]$} & \multirow{2}{*}{0.201} \\
\hline & Normal & $2(12.5)$ & $2(20.0)$ & & \\
\hline
\end{tabular}

have recommended the need for larger randomized controlled trials to prove the efficacy of intralipid before it can be recommended for routine use. In 2016, Dakhly et al demonstrated that Intralipid administration did not increase the frequency of chemical pregnancy (15). However, they recommended that findings related to ongoing pregnancy and live birth should be investigated further (15). They also defined the use of intralipid as a valuable therapy and advised that its use should be based solely on randomized controlled trial (16).

Needless to say, the present study is not without limitations. The main limitations are the small sample size, lack of the control group, and the retrospective study design.
A randomized controlled study with a larger number of patients is needed. More conclusive evidence in terms of the best dose and timing of intralipid for the patients with MIF is also required.

\section{Conflict of Interests}

Authors declare that they have no conflict of interests.

\section{Ethical Issues}

This study was approved by the institutional review board (IRB) of KFMC prior to starting (IRB 16-440, 08/12/2016).

\section{Financial Support}

None. 


\section{Acknowledgments}

The researchers would like to thank Mr. Mohammed Bashir for his help in statistical analysis part of study.

\section{References}

1. Simon A, Laufer N. Assessment and treatment of repeated implantation failure (RIF). J Assist Reprod Genet. 2012;29(11):1227-1239. doi:10.1007/s10815-012-9861-4

2. Chaouat G, Dubanchet S, Ledee N. Cytokines: Important for implantation? J Assist Reprod Genet. 2007;24(11):491505. doi:10.1007/s10815-007-9142-9

3. Sharkey A. Cytokines and implantation. Rev Reprod. 1998;3(1):52-61.

4. Sher G, Herbert C, Maassarani G, Jacobs MH. Assessment of the late proliferative phase endometrium by ultrasonography in patients undergoing in-vitro fertilization and embryo transfer (IVF/ET). Hum Reprod. 1991;6(2):232-237.

5. Ndukwe G. Recurrent embryo implantation failure after in vitro fertilization improved outcome following intralipid infusion in women with elevated $\mathrm{T}$ Helper 1 response HumFertil. 2011;14(2):21-22.

6. Roussev RG, Acacio B, Ng SC, Coulam CB. Duration of intralipid's suppressive effect on NK cell's functional activity. Am J Reprod Immunol. 2008;60(3):258-263. doi:10.1111/j.1600-0897.2008.00621.x

7. Kalfarentzos F, Kokkinis K, Leukaditi K, Maroulis J, Onoufriou A, Alexopoulos K. Comparison between two fat emulsions: Intralipid 30 cent vs intralipid 10 cent in critically ill patients. Clin Nutr. 1998;17(1):31-34. doi:10.1016/S0261-5614(98)80040-X

8. Kumar P, Mahajan S. Preimplantation and postimplantation therapy for the treatment of reproductive failure. J Hum Reprod Sci. 2013;6(2):88-92. doi:10.4103/0974-
1208.117165

9. Fatemi HM, Popovic-Todorovic B. Implantation in assisted reproduction: a look at endometrial receptivity. Reprod Biomed Online. 2013;27(5):530-538. doi:10.1016/j. rbmo.2013.05.018

10. El-khayat W, El Sadek M. Intralipid for repeated implantation failure (RIF): a randomized controlled trial. Fertil Steril. 2015;104(3):e26. doi:10.1016/j.fertnstert.2015.07.080

11. Clark DA. Intralipid as treatment for recurrent unexplained abortion? Am J Reprod Immunol. 1994;32(4):290-293.

12. Calder PC, Newsholme EA. Polyunsaturated fatty acids suppress human peripheral blood lymphocyte proliferation and interleukin-2 production. Clin Sci (Lond). 1992;82(6):695-700.

13. Abdolmohammadi-Vahid S, Danaii S, Hamdi K, Jadidi-Niaragh F, Ahmadi M, Yousefi M. Novel immunotherapeutic approaches for treatment of infertility. Biomed Pharmacother. 2016;84:1449-1459. doi:10.1016/j. biopha.2016.10.062

14. Check JH, Check DL. Intravenous intralipid therapy is not beneficial in having a live delivery in women aged 4042 years with a previous history of miscarriage or failure to conceive despite embryo transfer undergoing in vitro fertilization-embryo transfer. Clin Exp Obstet Gynecol. 2016;43(1):14-15.

15. Dakhly DMR, Bayoumi YA, Sharkawy M, et al.I ntralipid supplementation in women with recurrent spontaneous abortion and elevated levels of natural killer cells. Int J Gynaecol Obstet. 2016;135(3):324-327. doi:10.1016/j. ijgo.2016.06.026

16. Shirlow RH, Healey M. The effect of intralipid on pregnancy rates in in vitro fertilisation (IVF). Fertil Steril. 2016;106(3): e337-e338. doi: 10.1016/j.fertnstert.2016.07.956.

(C) 2018 The Author (s); This is an open-access article distributed under the terms of the Creative Commons Attribution License (http://creativecommons.org/licenses/by/4.0), which permits unrestricted use, distribution, and reproduction in any medium, provided the original work is properly cited. 